\title{
Control of Orientation Response by Auditory Discriminative Stimuli in the Carp
}

\author{
Ichiro Aoki*1 \\ (Accepted March 11, 1987)
}

\begin{abstract}
Acquisition of auditory discrimination and orientation response in carps was investigated under several reinforcement conditions. In the same-side condition, the sound-producing speaker and the reinforcer of food or electric shock were adjacent. The fish in this condition learned to obtain food by swimming toward the sound-producing speaker and to avoid electric shock by swimming in the direction away from the sound-producing speaker. In the opposite-side condition, the reinforcer of food or electric shock was opposite to the active speaker. The fish in this condition had a great difficulty in acquiring acoustic orientation, though the rate of learning in the food reinforced initial training and "go-no go" discrimation was similar to that for the same-side group. Another experiment, in which fish were trained to approach the correct feeding place by discriminating two kinds of the sound signal, showed that the fish was able to associate the two sound signals with the respective correct feeding place. These results were discussed in relation to utilization of cues in the orientation response in carp.
\end{abstract}

It is now clear that fishes can locate sound sources in the acoustic near- and far-field. ${ }^{1-3)}$ In addition to detecting and discriminating sounds, it is of considerable importance for fishes to sense the direction of sound-emitters in the environment in utilizing sounds for their survival. Directional information enables fishes to get food, to avoid predators and to meet mates. It has been considered that the ability to locate a sound source has acted as selective pressures on fishes. ${ }^{1,23}$

Recent electrophysiological and electron microscopic studies have shown that directional hearing in fishes is most likely based on direct stimulation of the two ears by particle motion and comparison of the output of differently oriented hair cell groups in macula. $\left.{ }^{2}, 4\right)$

By using conditioning techniques and biological sounds, behavioural studies have shown that several teleosts and sharks can orient to and swim in the direction of a sound source. ${ }^{5-8)}$ In these behavioural experiments, the primary concern is with the determination of the sensory ability of sound localization. In contrast to them, the present experiments are designed to investigate behaviour patterns in acoustic orientation in carps by comparing acquisition of auditory discrimination under several reinforcement conditions. This paper reports the results of three experiments which show that the carp can or cannot readily learn to get food and to avoid danger depending on conditions of auditory stimulus and reinforcement.

\section{Materials and Methods}

Young carps Cyprinus carpio, about $11 \mathrm{~cm}$ in total length, were used in this study. They were obtained from a cultured-fish dealer. The experiment was conducted on shoals of four or five fish in a plastic tank, $200 \mathrm{~cm}$ long, $50 \mathrm{~cm}$ wide and $40 \mathrm{~cm}$ deep. The experimental apparatus is illustrated in Fig. 1.

An underwater speaker was suspended in each end part, partitioned by a net, of the experimental tank. In food conditioning, a remote-controlled feeder which provided a fixed amount of food pellets was mounted diagonally above each speaker. In avoidance conditioning, six pairs of electrodes of stainless steel rod which provided an electric shock were set at sides of the tank. Water in the tank was circulated and filtered by a pump and a filter box located beside the tank. The water from the pump flowed to the water surface in the middle part of the tank. The water temperature was kept at $24^{\circ} \mathrm{C}$. Training was started about $30 \mathrm{~min}$ after fish were put into the experimental tank. The fish were allowed to swim freely in the tank. The behaviour of the fish was 


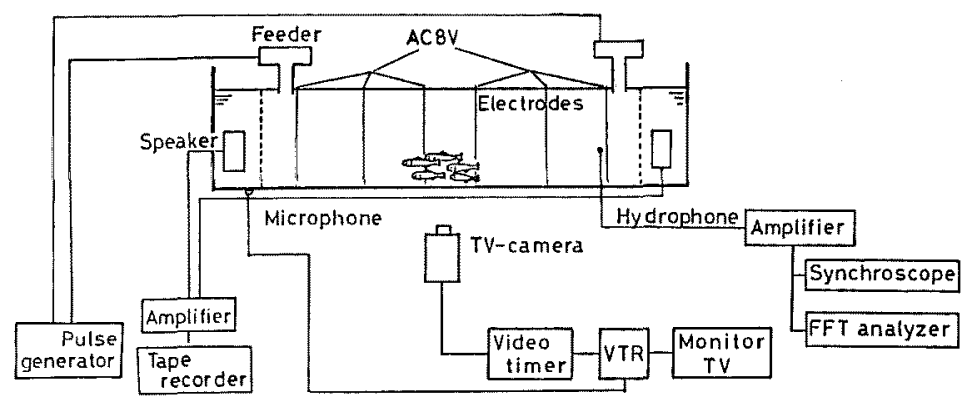

Fig. 1. Schematic diagram of the experimental apparatus.
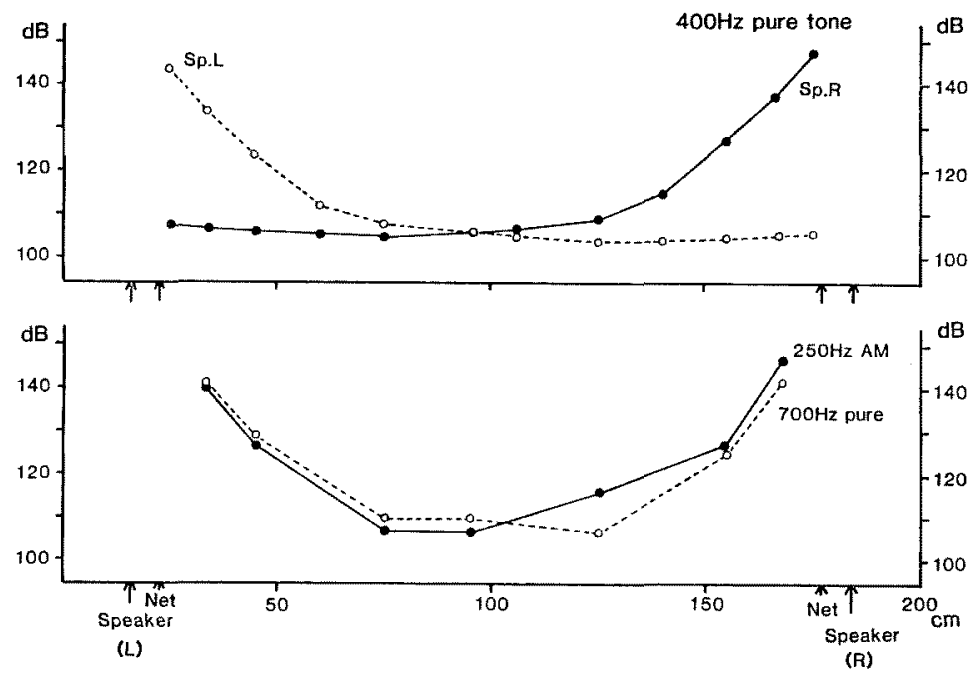

Fig. 2. Sound pressure $(0 \mathrm{~dB}=1 \mu \mathrm{Pa})$ in the experimental tank, measured on the sound Taxis. A $400 \mathrm{~Hz}$ pure tone was emitted by either the right (Sp. R) or left (Sp. L) speaker. A $700 \mathrm{~Hz}$ pure tone or a $250 \mathrm{~Hz}$ AM tone was emitted by both the right and left speakers.

monitored and recorded on video-tapes by means of a TV-camera.

Three kinds of sound stimuli were used: continuous pure tones of $400 \mathrm{~Hz}$ and $700 \mathrm{~Hz}$ and a sinusoidally amplitude-modulated (AM) tone having a $250 \mathrm{~Hz}$ carrier and a $30 \mathrm{~Hz}$ modulation rate. These tones were generated by an oscillator and recorded on tapes. Then, the sound signals were presented to the fish using a tape recorder, an amplifier and the underwater speaker. Sound pressure levels were measured with a hydrophone and a FFT-analyser. The sound intensity gradient on the sound axis is given in Fig. 2. Complex distributions of the sound intensity must have existed in the tank due to reflections by the wall and floor of the tank and the water surface. When the hydrophone was moved vertically and laterally from the measuring point shown in Fig. 2, the sound amplitude varied in a range of $\pm 10 \mathrm{~dB}$. However, since the acoustic conditions were campatible with the aim of this study, detailed mapping of the sound amplitude was omitted. Spectral levels of the background noise decreased with frequency and ranged from 85 to $65 \mathrm{~dB}$ at frequencies between 100 and 2500 $\mathrm{Hz}$, and were greater $(85-95 \mathrm{~dB})$ at frequencies between 2.5 and $6 \mathrm{kHz}$.

This study consists of three types of experiments (Experiments 1, 2 and 3) in which combinations of conditioned stimulus (CS) and unconditioned stimulus (UCS) differed from each other. The experimental conditions used with each shoal are given in Table 1.

In each Experiment, conditioning procedures were divided into three steps: Step I (initial training), Step II ("go-no go" discrimination) and Step III (acoustic orientation). However, durations of each step were different in shoals, and fish were 
Table 1. Experimental conditions

\begin{tabular}{|c|c|c|c|c|c|}
\hline Experiment & $\begin{array}{c}\text { Conditioned } \\
\text { sound stimulus }\end{array}$ & $\begin{array}{c}\text { Unconditioned } \\
\text { stimulus }\end{array}$ & $\begin{array}{c}\text { Same (S) or } \\
\text { Opposite (O) side }\end{array}$ & Shoal & $\begin{array}{l}\text { Training step and } \\
\text { its duration (days) }\end{array}$ \\
\hline \multirow[t]{7}{*}{1} & \multirow{7}{*}{$\begin{array}{c}\text { continuous } 400 \mathrm{~Hz} \\
\text { pure tone }\end{array}$} & \multirow[t]{7}{*}{ food } & $\mathbf{S}$ & $\mathrm{G} 1\left(4^{*}\right)$ & III (29) \\
\hline & & & S & No. $1(5)$ & I (10) II (16) \\
\hline & & & $\mathbf{S}$ & No. $3(5)$ & I (10) II (2) III (13) \\
\hline & & & $\mathbf{S}$ & No. $4(5)$ & I (10) II (2) III (13) \\
\hline & & & $\mathrm{O}$ & G2 (4) & III (30) \\
\hline & & & $\mathrm{O}$ & No. $2(5)$ & I (10) II (16) III (15) \\
\hline & & & $\mathrm{O}$ & $\mathrm{N} 2(5)$ & I (12) II (2) III (42) \\
\hline \multirow[t]{2}{*}{2} & continuous $700 \mathrm{~Hz}$ & \multirow[t]{2}{*}{ food } & & No. 5(5) & I (11) II (29) III (13) \\
\hline & pure or $250 \mathrm{~Hz} \mathrm{AM}$ tone & & & No. $6(5)$ & I (10) II (2) III (23) \\
\hline \multirow[t]{3}{*}{3} & continuous $400 \mathrm{~Hz}$ & \multirow{3}{*}{$\begin{array}{l}\text { electric } \\
\text { shock }\end{array}$} & $\mathbf{S}$ & E1 (5) & I (11) II (18) III (15) \\
\hline & pure tone & & $\mathrm{O}$ & E2 (5) & I (36) III (21) \\
\hline & & & $\mathrm{O}$ & E3 (5) & I (29) II (2) III (21) \\
\hline
\end{tabular}

* no. of fish in a shoal.

not always trained in the order of Steps I, II and III. For example, Shoal G1 was trained with a procedure in Step III from the beggining.

Details of conditioning and testing procedures are described below.

Experiment 1.

A continuous $400 \mathrm{~Hz}$ pure tone was presented as CS from either the right or left speaker, and a food reward was given as UCS from either the right or left feeder. There were two experimental conditions: the same-side condition in which food was presented at the same side as the active speaker and the opposite-side condition in which food was presented at the opposite side to the active speaker.

Step I. With the speaker and the feeder fixed to either the right or left side, fish were trained to react the sound signal by approaching the fixed feeding place. A trial consisted of the sound emitted for $50 \mathrm{sec}$ and food presented $40 \mathrm{sec}$ after the onset of the sound. The trial was repeated eight times per day at an interval of $4-6 \mathrm{~min}$. A mount of food pellets was limited to $2 \mathrm{~g}$ per day.

Step II. After the initial conditioned response was established, in four of eight trials in a day the other speaker opposite to one driven in Step I produced the sound for $50 \mathrm{sec}$ without food reinforcement. The reinforced and unreinforced trials occurred in the random order. The fish were trained to respond by approaching the feeding place when one speaker produced the sound signal and by not approaching the feeding place when the other speaker did it.

Step III. The fish displaying the acoustic orientation swam toward the correct feeding place cued by the position of the sound-emitting speaker.
Namely, the fish were trained to swim toward the sound-producing speaker in the same-side condition and to swim in the direction away from the sound-producing speaker in the opposite-side condition in order to obtain a food reward. Choice of the right or left speaker was determined randomly. At the early period of the training, food was presented and the sound signal terminated if any one fish arrived at the correct feeding place after the onset of the sound signal. Then, as the training progressed, even if the fish were staying at the correct feeding place, the time interval between the onset of the sound and the presentation of food was gradually extended within a limit of $40 \mathrm{sec}$. Last two trials in a day were test trials in which food was presented $40 \mathrm{sec}$ after the onset of the sound. In addition, last three days in Step III were test sessions consisting of eight test-trials.

\section{Experiment 2.}

Conditioning and testing procedures similar to those in Experiment 1 were followed, though a continuous $700 \mathrm{~Hz}$ pure tone or a $250 \mathrm{~Hz}$ AM tone was presented as CS from both the right and left speakers.

Step I. With the sound signal and the feeding place fixed to either of the two, the fish were trained like in Step I of Experiment 1.

Step II. Next, in four of eight trials in a day, the other sound signal unused in Step I was presented without reinforcement.

Step III. Conditioned acoustic orientation was to approach the correct feeding place by discriminating two kinds of the sound signals. Training and testing procedures were similar to those in Step III of Experiment 1. 


\section{Experiment 3.}

A continuous $400 \mathrm{~Hz}$ pure tone and an electric shock were presented as CS and UCS respectively. The sound signal was emitted by either the right or left speaker and the electric shock provided by an electric field of $\mathrm{AC} 8 \mathrm{~V}$ passed between either right or left three pairs of electrodes. In the same-side condition, the speaker driven and the electric field applied were at the same side, and in the opposite-side condition, they were opposite to each other.

Step I. With the active speaker and the electric field fixed to either the right or left, the fish were trained to respond to the sound in order to avoid the electric shock. The electric shock followed $20 \mathrm{sec}$ after the onset of the sound signal, and both the sound and shock continued within a limit of $30 \mathrm{sec}$ duration until all the fish in a shoal passed the halfway line of the tank to escape from the electric field. In an avoidance response made within $20 \mathrm{sec}$ of the beggining of the sound, the sound terminated at the time when all the fish in a shoal escaped. Training in one day consisted of eight trials at an interval of 4-6 min.

Step II. Next, in four of eight trials in a day, the other speaker opposite to one driven in Step I emitted the sound signal without electric shocks.

Step III. In this step, the fish were trained to swim away from the sound-producing speaker in the same-side condition and to swim toward the sound-producing speaker in the opposite-side condition. The speaker driven on a particular trial was chosen randomly.

\section{Results}

\section{Experiment 1.}

The conditioned food-getting response to the sound consisted of swimming with accelerated velocity at the onset of the sound, aggregating and staying under the feeder. The response sometimes included displaying the action of snatching food pellets even before the food was dropped. After the sound terminated and the fish finished taking food, they swam round in the tank with composure again.

By replaying the video-tape recordings, the number of fish in each of the right and left feeding places was counted every $5 \mathrm{sec}$ for the sound signal and inter-signal periods. In this counting, on the basis of preliminary observations on fish aggregating and feeding, the feeding place was defined as a space below the feeder between the net and $25 \mathrm{~cm}$ distance from it. The total number of fish in the feeding place was obtained by counting 7 times for $30 \mathrm{~s}$ period from 10 to $40 \mathrm{sec}$ after the onset of the sound before the food presentation. In addition, the mean number of fish per $30 \mathrm{sec}$ period was calculated from the total number of fish in the feeding place for the overall inter-

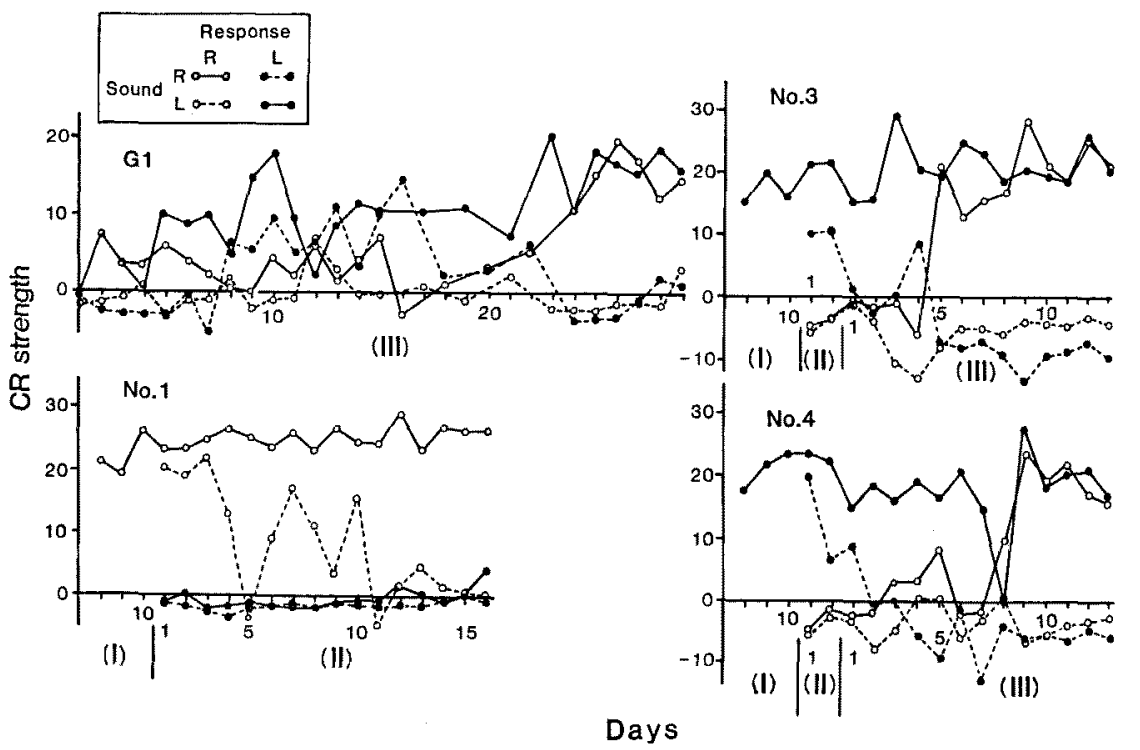

Fig. 3. Acquisition of the discrimination by the same-side group for which food was presented at the same side as the active speaker. The rightward or leftward response to the sound from the right or left speaker is plotted. Roman numerals indicate training steps. 
signal period between trials except $40 \mathrm{sec}$ period after the food presentation. Thus the conditioned response (CR) strength was represented by the difference between the total number of fish during the sound presentation and the mean number of fish during the inter-signal period. The measurement of the frequency of position recordings in the feeding place was made for all trials in Steps I and II and only for test trials in Step III.

Fig. 3 gives the mean CR strength per day for each shoal in the same-side condition. Shoals No. 3 and No. 4 showed a reliable discrimination and orientation response in the test sessions on the 11-13th days of Step III: the fish moved toward the sound-producing speaker and stayed in front of it. The performance level for each of the right and left speakers in the test sessions was similar to that in Step I. Although CR strengths in Step III except the test sessions were data from one trial for each of the right and left speakers, Nos. 3 and 4 appeared to reach the stable level on the 5th and the 9th day of Step III, respectively. Shoal G1, which was trained with the procedure of Step III from the beggining of conditioning, gave indications of the discrimination and orientation response on the 24th day. Then, it distinctly showed the acoustic orientation in the test sessions on the 27-29th days. Considering overall periods of conditioning, the rate of learning was slightly faster for Nos. 3 and 4 trained in the order of Steps I, II and III.

Shoal No. $1 \mathrm{kept}$ a stable level of performance of "go-no go" discrimination after the 11th day in Step II. During early periods of Step II, No. 1 moved not to the left corner but to the right corner as trained in Step I, when the left speaker was driven. Such a stimulus generalization was observed in Nos. 3 and 4 in Step II.

The learning curves for each shoal of oppositeside group are shown in Fig. 4. The results were quite different from those of the same-side group. On the 28-30th days G2 did not yet show the correct response of swimming toward the feeding place opposite to the sound-producing speaker. In both the initial training in Step I and the "gono go" discrimination in Step II, the rate of learning for No. 2 was similar to those for the same-side group. However, in Step III, No. 2 gradually attenuated the correct response established already, and eventually showed CR strengths of about 0 in the test sessions on the 13-15 days. At this time the fish seemed to get
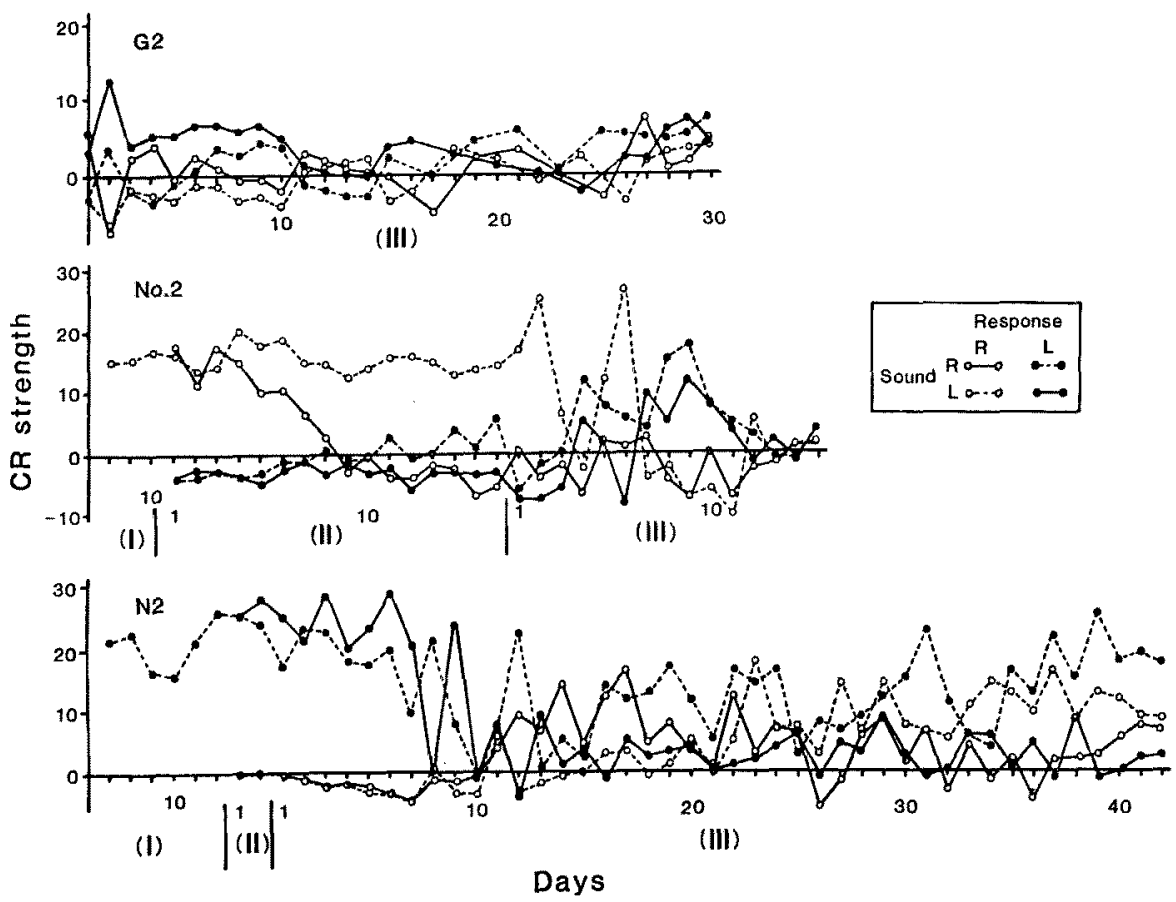

Fig. 4. Results of training for the opposite-side group for which food was presented at the opposite side to the active speaker. The rightward or leftward response to the sound from the right or left speaker is plotted. Roman numerals indicate training steps. 


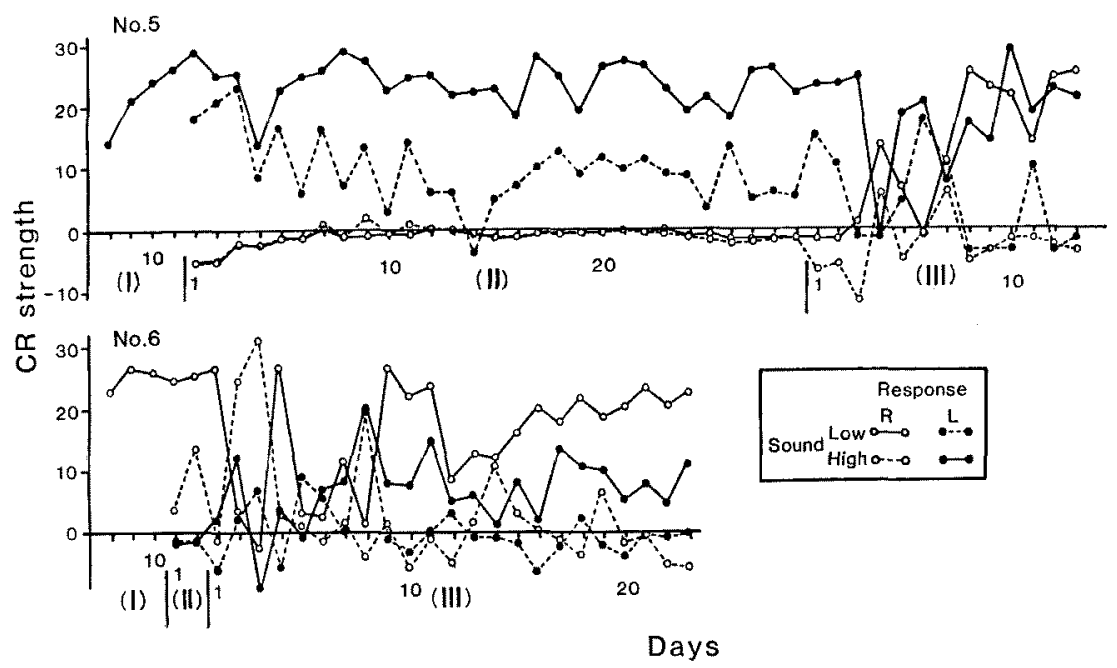

Fig. 5. Acquisition of the discrimination by each shoal in Experiment 2 in which food was presented at the left corner when the high frequency sound was emitted and at the right corner when the low one was emitted. The rightward or leftward response to the sound of the high or low frequency is plotted. Roman numerals indicate training steps.

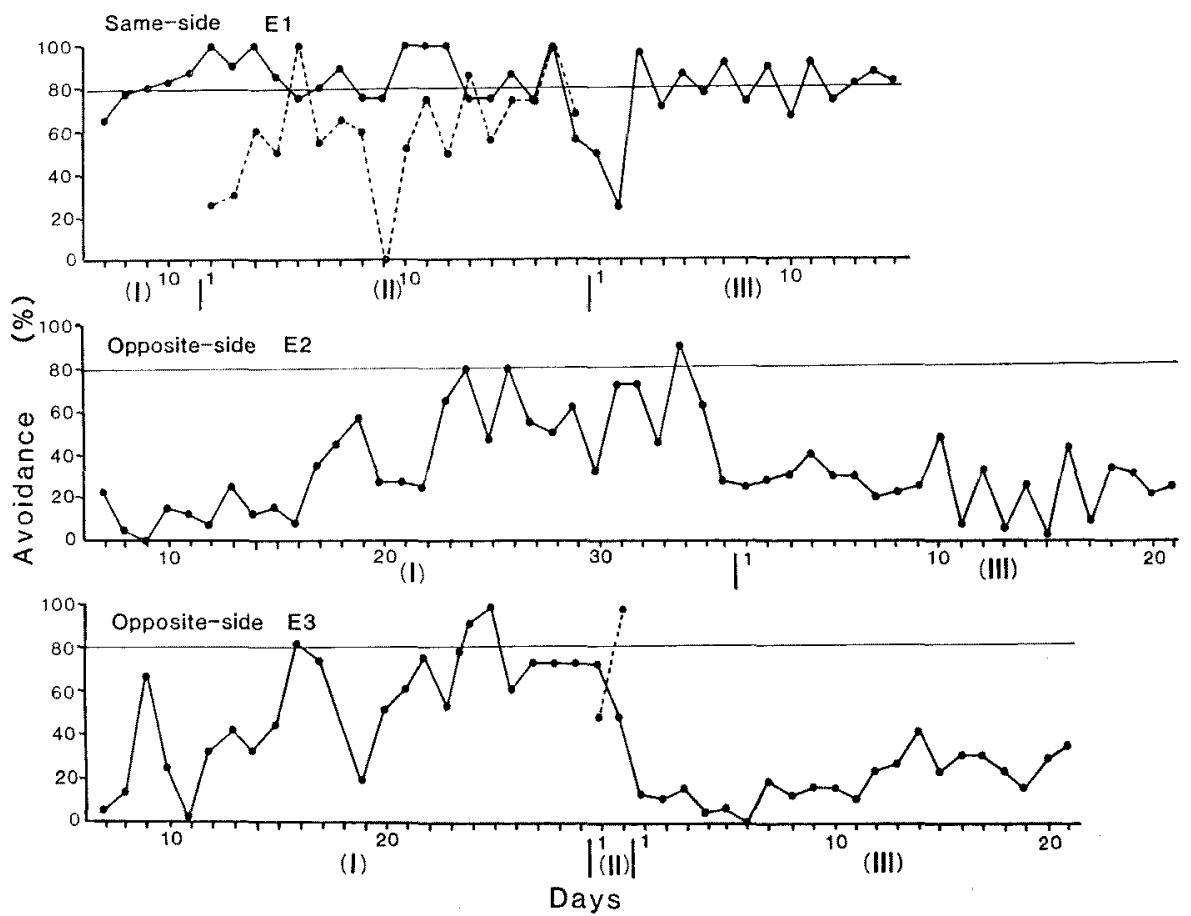

Fig. 6. Avoidance percentage for each shoal in the same-side and the opposite-side condition in Experiment 3. Broken lines indicate the percentage of incorrect "go" response in the go-no go discrimination in Step II. In Step III, the percentage was obtained with both stimuli combined. 
perplexed which was the correct feeding place, shuttling between the right and left corners in rapid succesion. N2 was trained for a long period of 42 days in Step III (Fig. 4, bottom). The correct response established in Step I was reduced in the same manner as in No. 2, and unstable responses were observed from the 10th to 34th day of Step III. In test sessions on the 40-42 days, though the correct responses occurred more frequently than the incorrect ones, the latter was still over a level of 0 . Learning of acoustic orientation was very difficult for fish in the opposite-side condition.

\section{Experiment 2.}

CR strengths were obtained in the same way as in Experiment 1. Fig. 5 shows the mean CR strength per day for each shoal. The "go-no go" discrimination by No. 5 was yet incomplete on the last 29th day of Step II. Then, in Step III, signs of the correct orientation response came on the 8th day and the performances for each of the high and low frequency sounds were as good as that of the correct response in Steps I and II.

Though Shoal No. 6 once showed a confusion of responses in Step III, they gave first indications of the correct response on the 9th day. In test sessions on the 21-23rd days, the performance level for the low sound was as high as seen in Step I, but that for the high sound remained low. Preferences of the high and low sounds and a kind of the preceeding effect may be related with this result. In this experiment, the fish was able to associate each of two sound signals with the correct feeding place.

\section{Experiment 3.}

The measure of avoidance learning was the percentage of those individuals in all trials of a day which avoided the electric shock by swimming into the half region of the tank in safety within $20 \mathrm{sec}$ of the beggining of the sound. The acquisition criterion was that the percentage remained more than $80 \%$ for 3 succesive days. The percentages of avoidance response per day are shown for the same-side and the opposite-side group in Fig. 6. Though the "go-no go" discrimination by E1 was obscure, the correct response tended to be higher percentage than the incorrect one. Then, in Step III, E1 reached the acquisition criterion on the 15th day.

The opposite-side E2 and E3 were trained for 36 and 29 days respectively in Step I, but the training resulted in no attainment of the criterion though the avoidance percentage over $80 \%$ sporadically occurred. Then, both Shoals exhibited no signs of acquisition with constant poor performance for $\mathbf{2 1}$ days of Step III. It is concluded that carps can learn to avoid the shock by swimming in a direction away from the sound source, and that they cannot easily learn to do so by swimming toward the sound source.

\section{Discussion}

In the present experiments, the acoustic field produced by the underwater speaker had complex patterns of reflection and interference and included both the near- and far-fields. The fish were allowed to swim freely in these acoustics of the tank. In such a situation, behaviours of arriving at and getting away from a sound source are based on both directional hearing and intensity gradient seeking. As a sensory organ the lateral line was probably involved in the behaviours as well as the inner ears. The dual sensory and behavioural mechanisms must function also in the movement of fish with respect to a sound source in natural environments. Although directional hearing is a more effective and important way of locating a sound source, ${ }^{5)}$ it is considered that fish operate suitably those different mechanisms depending on the distance from a sound source, the quality of a sound and the ambient acoustic conditions. ${ }^{10)}$

The opposite-side group in Experiment 1 acquired the initial food-getting response in Step 1 and the "go-no go" discrimination cued by the location of the sound source in Step III at a rate similar to the same-side group, but it had a great difficulty in acquiring the acoustic orientation in Step III. The stimulus generalization observed at the early period of Step II in the same-side and opposite side groups indicates that while the initial food-getting response in Step I was elicited by the sound, the direction of the movement was connected with the fixed feeding place by the visual cues and spatial memory not with the sound. Namely, the eliciting stimulus and the orientating stimulus of the conditioned response were two different things in the initial training. In Step II, since the time for food presentation was connected with the location of the sound source and the place for food was still connected with the definite place itself not with the sound, the "go-no go" discrimination was possible for the oppositeside group also. In Step III, however, the fish must of necessity associate the location of the sound source with the correct feeding place in order to obtain food. This learning depended 
on the positional relation between the location of the sound source and the feeding place. Food conditioning required that the sound source and the reinforcer of food should be adjacent or in the same direction. For the fish in the oppositeside condition the correct response for getting food was to swim in the direction in which the sound intensity decreased. It was very difficult for the fish to learn. Rats, one of higher vertebrates, also have been observed to have such a tendency in an auditory localization discrimination. ${ }^{11}$

The results of the avoidance conditioning also show that the sound source and the reinforcer of electric shock should be adjacent or in the same direction though the direction of movement relative to the sound source is reverse to that in the food conditioning. In nature, when an animal detect presences of its food and predators by sounds, the food and the predators usually exist at the sound source. Therefore, it is reasonable that the rinforcement produced good effect on the formation of the acoustic orientation if the reinforcer and the sound source are adjacent.

There are reports that squirrelfishes and gobies swam toward a speaker emitting conspecific alarm calls produced in response to predators. ${ }^{\text {? }}$ In the present experiment, it was very difficult to train the fish to swim toward the sound source using the electric shock. This behavioural difference indicates that the sound stimulus associated with the electric shock and the alarm calls are different in meaning. While the conditioned sound stimulus portends danger, it can hardly be a signal which shows that the location of the sound source is safe. On the other hand, it can be considered that the alarm calls, in addition to showing danger to mates, show that the location of the sound source is safe or lead to form a compact school by attracting mates in defence.

Following explanations are possible for the results of Experiment 2 that the fish were able to be trained to swim toward the correct feeding place at the right or left corner according to the sound frequency. In Step III, though the sound frequency showed the fish which was the correct feeding place, the sound was not a cue that directly guided them there unlike in Step III of Experiments 1 and 3. The fish associated the sound frequency with the place to swim toward in such a way that, for example, the high sound was associated with this place and the low sound with that place. In their orientation to the correct feeding place, the fish depended on the visual cues and spatial memory also in Step III like in Steps I and II of Experiment 1.

The results of the present experiments suggest that carp tends to use other cues such as visual ones than acoustic ones in its orientation when the former cues are available. This tendency may be a feature of carps or other freshwater fishes in the use of orientation cues. Inoue et al. ${ }^{12,13)}$ suggested that freshwater fishes tend to conduct the upstream movement depending on vision, and that, on the other hand, pelagic fishes do so depending on mechanical senses, for example the lateral line. In general, freshwater fishes have available visual objects such as stones and aquatic plants in their habitats, but pelagic fishes scarcely have any visual objects in their habitats. The same experiments on a pelagic fish as in this study may bring different results that the pelagic fish use the sound as orientation cues in Step I of Experiment 1. If so, it will encounter difficulty in the acquisition in the opposite-side condition since Step I of Experiment 1 and be hardly able to accomplish the task in Experiment 2.

Most of studies on conditioned responses of fishes have been conducted for sensory physiological purpose. The learning ability of an animal, however, has an adaptive significance, being one of products of its evolutionary process. ${ }^{14-18)}$ Therefore, an approach is valid to the behaviour and ecology of a fish by analysing what it can and cannot readily learn.

\section{References}

1) R. R. Fay: in "The Behavior of Fish and Other Aquatic Animals" (ed. by D. I. Mostofsky), Academic Press, New York, 1978, pp. 197-236.

2) A. N. Popper and S. Coombs: Am. Sci., 68, 429440 (1980).

3) A. D. Hawkins: in "Hearing and Sound Communication in Fishes" (ed. by W. N. Tavolga, A. N. Popper and R. R. Fay), Springer-Verlag, New York, 1981, pp. 109-137.

4) A.D. Hawkins: in "The Behaviour of Teleost Fishes" (ed. by T.J. Pitcher), Croom Helm, London, 1986, pp. 114-151.

5) D. R. Nelson: Bull. Mar. Sci., 17, 741-768 (1967).

6) A. A. Myrberg, Jr.: in "Behavior of Marine Animals, Vol. 2" (ed. by H. E. Winn and B.L. Olla), Plenum Press, New York, 1972, pp. 435468.

7) A. N. Popper, M. Salmon, and A. Parvulescu: Anim. Behav., 31, 86-97 (1973).

8) A. Schuijf and M. E. Siemelink: Experientia, 
30, 773-775 (1974).

9) A. Schuijf, C. Visser, A. F. M. Willers, and R. J. A. Buwalda: Experientia, 33, 1062-1063 (1977).

10) H. Abe: The Study on Hearing in Fish. D. Thesis, Univ. of Tokyo, 1976, pp. 79-95.

11) M. D. Beecher and J. M. Harrison: J.exp. Anal. Behav., 16, 193-199 (1971).

12) M. Inoue, W. G. Ning, and T. Arimoto: Nippon Suisan Gakkaishi, 48, 1697-1701 (1982).
13) M. Inoue, T. Arimoto, and W. G. Ning: Nippon Suisan Gakkaishi, 50, 63-69 (1984).

14) N. Tinbergen: The Study of Instinct (Japanese transl. by T. Nagano), Sankyo Shuppan, Tokyo, 1975, pp. 142-147.

15) H. C. Wilcoxon, W. B. Dragoin, and P. A. Kral: Science, 171, 826-828 (1971).

16) R. C. Bolles: Learning Theory (Japanese transl. by H. Imada), Baifukan, Tokyo, 1982, pp. 246-250. 